AlII05 476 395

NLT

NISTIR 6180
NIST

PUELICATIONS

U.S. Department of Commerce

National Institute of Standards and Technology

High Performance Systems and Services Division

Scalable Parallel Systems and Applications Group

\title{
User Guide to CADMUS, a Simplified Parallel Code
}

for Laplacian-fractal Growth

Howland A. Fowler, Judith E. Devaney, and John G. Hagedorn

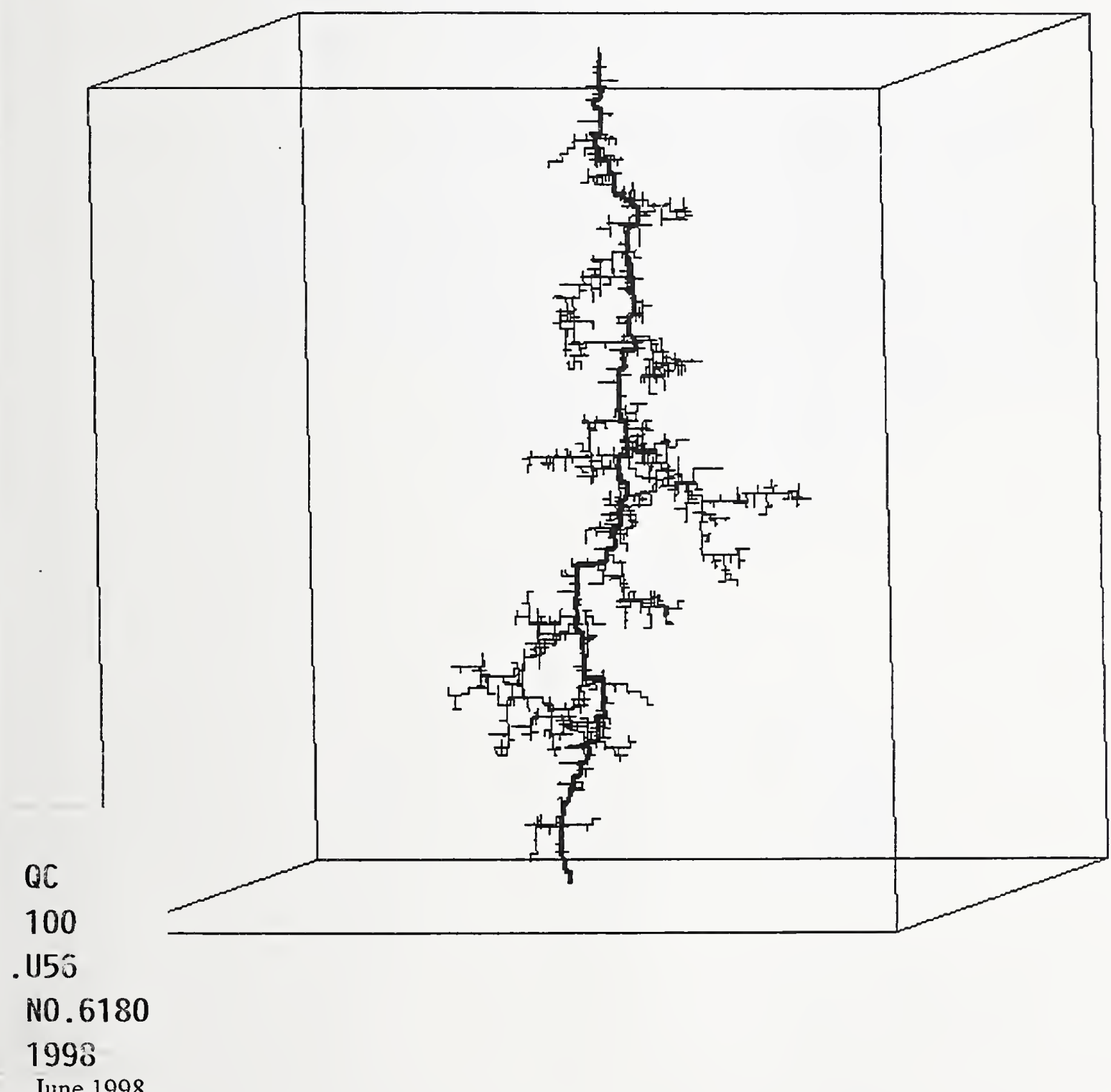

June 1998 



\section{NISTIR 6180}

\section{User Guide to CADMUS, a Simplified Parallel Code for Laplacian-fractal Growth}

\section{Howland A. Fowler Judith E. Devaney John G. Hagedorn}

U.S. DEPARTMENT OF COMMERCE Technology Administration National Institute of Standards and Technology

High Performance Systems and Services Division, ITL Gaithersburg, MD 20899-0001

June 1998

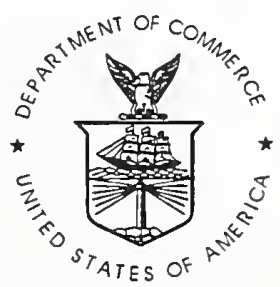

U.S. DEPARTMENT OF COMMERCE William M. Daley, Secretary

TECHNOLOGY ADMINISTRATION

Gary R. Bachula, Acting Under Secretary for Technology

NATIONAL INSTITUTE OF STANDARDS

AND TECHNOLOGY

Raymond G. Kammer, Director 



\title{
User guide to CADMUS, a simplified parallel code for Laplacian-fractal growth
}

\author{
Howland A. Fowler, Judith E. Devaney and John G. Hagedorn \\ High performance Systems and Services Division, ITL \\ National Institute of Standards and Technology \\ Gaithersburg, MD 20899
}

\begin{abstract}
High-voltage breakdown in liquid dielectric is simulated as growth of a stochastic Laplacian fractal. The model is contained in a software package that is written in Fortran 90 with data parallel extensions for distributed execution. These extensions encapsulate an underlying, invisible message-passing environment (MPI), thus enabling the solution of memory intensive problems on a group of limited-memory processors. Block-partitioning creates processes of reasonable size, which operate in parallel like small copies of the original code. The user needs only to express his model in transparent array-directed commands; parallel interfacing between blocks is handled invisibly. Breakdown is performed in parallel, in each of the local blocks.
\end{abstract}

Contribution of NIST, not subject to copyright in the U.S.

* Guest researcher 


\section{Introduction}

This model simulates the growth of high-speed filamentary streamers, during high-voltage breakdown in liquid dielectrics.

Fortran 90 is used as a high-level parallel language for the code, supplemented by NIST's DPARLIB, a set of subroutines which extend F90 across block-process boundaries, providing an invisible interface to the Message Passing Interface (MPI).

Fortran 90's advantages are

- addressing huge arrays directly, so as to take advantage of the large individual-processor memory which is currently available on workstations and multiprocessors.

- allowing the assembly of program logic with combinations of real and logical arrays.

- it contains the powerful WHERE mask, CSHIFT, PACK and UNPACK, distributing and collecting operations.

- user needs to loop over arrays only when reading in and out.

- organization of the program into modules makes for easy substitution, compiling, and testing of code.

DPARLIB's extensions permit

- carrying F90 parallelism across block partitioning.

- taking advantage of MPI's ability to facilitate and extend C-shifts based upon a Cartesian-grid topology.

- The underlying MPI code is completely hidden; the resulting program is very similar to serial code; indeed, it is so executed by the individual processor.

- All processes start the same instructions together.

- A program may be built and tested on one processor, then scaled upward to execute over a 2-D array of process blocks.

Thus, we have a high-level scalable language: ***** Fortran 90 / DPARLIB / MPI ***** in which it is easy to model physics problems, test the (serial) code at small scale, then enlarge to full scale on a multiprocessor 
(for example IBM SP2, SGI Onyx, SGI Origin ${ }^{1}$ ). The language is particularly well-suited to problems which are easily expressed on a large Cartesian grid, since MPI is adapted for communicating data across rectangular block boundaries.

The model is a routine for the stochastic growth of Laplacian fractals on large 3-D Cartesian grids [1]. While the intended application has been to simulate global features of the growth of fast streamers in liquid dielectrics, the family of possible application areas is larger than this ( water-treeing in solid dielectrics, growth of metallic structures in electrodeposition, surface streamer-spreading at dielectric interfaces are examples ). Each would require some specialization of parameters and boundary conditions. These routines are presented as expository examples.

The elements of the algorithm are:

1. Assume streamer tree fully conductive, and attached to the anode electrically.

2. Solve Laplace's equation throughout the interior region, using the anode and streamer tree as one boundary, and the cathode as the counterelectrode boundary.

3. Examine neighbor sites to the tree. If phi is above threshold (cutoff) level, then compare against weighted random numbers. If they exceed, attach to tree.

4. Cycle until counter-electrode is reached.

Adjustable parameters of the calculation (grid bounds, needle position, threshold (cutoff) voltage level, and power-law exponent) allow a broad range of fractal behavior to be approximated.

Novel features of the realization include:

- Concurrent (simultaneous) growth is distributed over the entire tree at any instant

- Simulated time progression (Monte-Carlo time ticks) is recorded. When time-compression is used to avoid empty statistical trials, the MonteCarlo time is estimated.

\footnotetext{
${ }^{1}$ Certain commercial equipment, instruments, or materials are identified in the paper to foster understanding. Such identification does not imply recommendation of endorsement by the National Institute of Standards and Technology, nor does it imply that the materials or equipment identified are necessarily the best available for the purpose.
} 
- Full three-dimensional display of the growth process (animated or color-banded) is possible.

- Compact procedures are used for statistical testing and for time compression.

- Face- and body-diagonal links may be included in the fractal tree.

The code is presented as a directory of modules (cadmus/source), see Figure 1, which are compiled by Makefile under four separate main programs (cadone, cadtwo, cadmus, cadbkl), each of which performs a different task. cadone makes a comparison against a linear-weighted uniform random-number distribution, and counts the number of statistical tries (Monte Carlo time ticks). cadtwo uses a square-law-weighted randomnumber distribution, and counts statistical tries. cadmus and cadbkl allow a choice of integer exponents for the weighting. In these latter two programs, a normalization is used which corresponds to time compression for low probabilities, PACKing and weighting the neighbor-voltage distribution, and then comparing against a uniform random number distribution, compressed when the voltage values are low (as at the outset). In such cases we estimate the progression of statistical tries, instead of counting. This permits cube-law and higher powers to be tested, without a very large number of "empty" statistical tries. It has proved useful for tracing the fractal shape of cube-law and fourth-power trees. cadbkl contains an estimating formula for the value of Monte-Carlo waiting time between events (important when the field strengths and discharge probabilities are low) as presented by I. Beichl and F.E. Sullivan [2]. [The formula used in cadmus routine is noticeably less accurate for this estimation.]

The directory cadmus/diag contains modified versions of the cadone and cadtwo routines, which permit the addition of face- and body-diagonal links in the tree growth. The greatly increased choice of directions from each site, at each step of growth, leads to denser growth and more irregular patterns of stems.

The routines have been run on both multiprocessors (IBM SP2, SGI Onyx, SGI Origin) and open clusters of SGI workstations - sometimes referred to as networks of workstations (NOWs) - linked by LAM, with access to a common memory-server.

For grid sizes up to $64 \mathrm{X} 64 \mathrm{X} 64$, workstation clusters or running on a time-shared Onyx are satisfactory for development work, with interactive runs. For large grid sizes, namely $100 \times 100 \times 100$ or $128 \times 128 \times 128$, the limitations are set by the burden of repetitive computation of Laplacian 
convergence. The number of available dedicated processors will determine the duration of the-run, and will have to be determined by the local batch queueing protocol.

Program Requirements:

Cadmus routines requirexs both MPI and the DPARLIB library. On an SP2, MPI can be the IBM Parallel Operating Environment (POE) MPL or a public-domain MPI like LAM [3] or MPICH [4]. On an SGI, MPI can be SGI's MPI or a public-domain MPI. DPARLIB can be obtained from our web site:

http://www.itl.nist.gov/div895/sasg

\section{Details of the Cadmus Routines}

Cadmus, after slaying a dragon, was instructed to sow its teeth on the ground. There sprang up an array of fully armed soldiers, who fought among themselves until only five remained...

$* * * * * * * * * * * * * * * * * * * * * * * * * * * * * * * * * * * * * * * * * * * * * * * * * * * * * * * * *$

The main program cadmus calls routines from the other modules. These are compiled in the Makefile, in the order in which the main requires them. Note that the Makefile needs object code for DPARLIB and MPI to be in place, in order to compile, and may need to be modified to state the correct DPARLIB location on the local system. In the listings below, modules also carry the suffix [underscore plus mod].

MAIN programs:

cadmus - calls fast3;

cadbkl - calls fastbkl;

cadone - calls singlcomp;

cadtwo - calls dblcomp.

Setup, initializing modules:

globals; breakup; growinit.

Display modules, printing to screen:

treedisp; nbrdisp. 
Program logic and arithmetic:

nbr; lpl; fast3; fastbkl; singlcomp; dblcomp.

The modules contain data, assignments, and subroutines as follows:

globals reads from the input file 'breakdown.in' the integers decribing the desired array of process blocks, and the dimensions of the desired rectangular grid (the latter must be an even number in the c- or z-direction, along which the blocks are partitioned. The global z-dimension, divided by the number of process blocks, must also be an even number, in order for the red and black subgrids to match up at the boundaries.) It also contains a list of 32 seed integers for the random number generator dp-uni. In order to create a new run, alter the lead integers in the list and recompile. Only as many seed integers will be used, as number of processes.

breakup allocates major arrays within the bounds of the individual process blocks. It also contains a routine to assign the red and black subgrids.

growinit starts with the subroutine gradient, which fills the interior volume with a linear-gradient voltage field 'phi'. This is a zeroth approximation which reduces later convergence time. It then allocates and initializes the 'tree' array, including the anode plate and the concentrating needle. It allocates and initializes the cathode plate. It runs a test round-robin access sequence to the output file streamer (if the empty streamer file is not present, the program will stop). The subroutine writestream will be used for output.

treedisp contains a test routine for displaying to screen one plane of the 'tree' array. When used, it displays, successively, all the planes in one of the center-most blocks of the calculation, with +'s and .'s to mark the disharged and empty sites. (Check to make sure that the starting needle is located within the appropriate block.)

nbr finds all neighbor positions which are one grid step away from the tree array as then constituted, identifying possible candidates for breakdown in the next statistical try.

nbrdisp contains a test routine for displaying one plane of the nbrs array. As above, it may need adjustment before use.

lpl contains the subroutine laplace which does the major repetitive calculation of the voltage field, by Gauss-Seidel over-relaxation. Epsilon is set at 0.0050 ; but this may be changed. Setting epsilon to 0.0010 will roughly double the number of convergence loops to reach completion. Also here is subroutine lplinit for initialization; this is set to run for 200 loops instead of using the epsilon. 
fast3 describes the statistically-weighted growth stage. This is the statistical-choice routine for the cadmus main. (The main action alternates between fastthree and laplace.) fastthree identifies neighbor candidates whose voltages exceed the 'cutoff' threshold, PACKs their values on 'philin' array, exponentiates that to 'pwr' onto 'phisq' linear array, and sums the elements of 'phisq' to obtain the a-priori expected probability of any event occurring. This sum is used to weight a uniform random distribution, for test comparison against 'phisq'. Survivors are UNPACKed to their original positions, forming the array 'nutree', which is combined with 'tree' as the growth step.

fastbkl differs from fast3 in utilizing the direct BKL approximations for estimating the number of "empty trials" to reach a composite probability of one [2]. Where fast3 tends to overstate this quantity, when the probabilities are very low, fastbkl may do a better job.

The Dparlib random number generator is used; it starts from a separate seed within each process and creates independent streams of random numbers.

Both laplace and fastthree terminate with global-count if loops which ensure that the step into the alternate subroutine is performed simultaneously in all processes.

Output into the 'streamer' file is a five-column list; three position integers followed by the breakdown voltage at that site and the statistical trial number (Monte Carlo time tick).

singlcomp is the statistical-choice routine for the cadone main program. It differs from fast3 in counting all statistical trials, instead of just estimating the ones with low probability. No PACK routine is called. The neighbor phis are compared against a uniform distribution of random numbers, at their sites.

dblcomp is the corresponding routine for the cadtwo main program. Instead of exponentiating the field to power 2 , as in fast3, it finds a MAX array from two linear arrays, each filled with a uniform distribution of random numbers. This is equivalent to conditional(or product) combination, passing the distribution of voltages at the neighbor positions over a "double hurdle" of filters, and leaving a square-weighted distribution of survivors.

cadone and cadtwo are set up to call nbrdisp and treedisp at convenient intervals during an interactive run. cadmus instead prints a lot of numerical information at the steps of the statistical routine, which can be read to tell the status of the run. When not desired, these printout instructions may be commented out; or, their output may be led to a null file. 
IMPORTANT NOTE: USE CADBKL WHEN THE DESIRED (SURVIVOR) POWER-LAW EXPONENT IS 3 OR HIGHER, IN ORDER TO AVOID MANY EMPTY STATISTICAL TRIAL LOOPS WHEN THE FIELD STRENGTHS ARE SMALL.

\section{Input required from user}

The program looks for an input file 'breakdown.in' which must contain six lines of integers:

Line 1 gives the global upper bounds of the grid, in three columns

Line 2 gives the 2-D array configuration of process blocks

Lines 3,4 and 5 give the global bounds of the anode needle: $\mathrm{X}$ or a direction in line 3 is direction of growth,anode to cathode. $\mathrm{Y}$ or b direction, line 4 , is orthogonal to this, across the growth axis. $\mathrm{Z}$ or $\mathrm{c}$ direction, line 5 , is also orthogonal; this is the direction in which the process blocks are stacked.

Line 6 gives the desired exponent for the statistical weighting of the power law. It is read by cadbkl and cadmus, and is ignored by cadone and cadtwo.

\section{Guide to Operating}

Before starting each run, make sure that an empty file 'streamer' exists, to catch the listing of breakdown sites from the individual processes. The unix commands "rm streamer" followed by "touch streamer" will do this job. In its present realization, the programs assume that all processes will have access (round-robin) into a common file; this is especially convenient if the run happens to be interrupted, because it will contain the full growth history of the streamer, up to that moment.

If the processes cannot communicate to a common file, then the output instructions should be adjusted to form a streamer file for each process, which will dump at the end into a common 'streamer'. This should then be sorted by statistical trial number (Monte Carlo time tick) to arrive at the correct sequence.

If a new selection of random-number seeds is desired, change the leading integers in the data list of 32 seeds, which is supplied in globals-mod.f90, and recompile. Be sure that there are 32 in the list after you modify it.

If a different value of cutoff (threshold phi) is desired, change the assigned value of this parameter at the beginning of fast3 (or fastbkl, singlcomp, 
dblcomp) and recompile.

If a value of convergence epsilon in the Laplacian calculation, other than 0.0050 is desired, change this assigned value at the start of subroutine laplace, in lpl, and recompile.

Subroutines showtree and shownbrs were used in early testing of the program on small grids, say $48 \times 48 \times 48$. To use shownbrs the integer $\mathrm{k}$ is set to the z-plane in which the anode needle is located (usually identified with the central-most process block), and the routines produce a screen display of +'s and .'s, to indicate the subarray of tree or nbr sites in that plane only. As a general rule, they are not needed in larger runs - the system will print (to screen) enough data to show the progress of the run.

\section{Graphics for display of the breakdown tree}

The 5-column 'streamer' list file generated by Cadmus runs must be postprocessed to render it in 3-D graphical format. See Figure 2.

Three programs are used: nuldrread prepares two files, 'newtree' and 'ldr', which can be read in black and white line display (fixed angle) by PVWave graphics routines, several examples of which are provided, having the suffix .pvw. For a system provided with PVWave support, the calls are then "wave", and ".RUN xxx.pvw", where xxx stands for the name of the specific PVwave program.

nuldrread makes a backward search to find the link connections implied by the 'streamer' listing, and then lists all of these links. Thereupon, it traces back through the array of the links to find the continuous path from cathode to anode, which it calls in a heavy-weight line display. The PVWave routines read the links and the continous path from the newtree and ldr files. In order for this leader-trace function to proceed correctly, the discharge of the site in the plane next to the cathode must be the last line of the 'streamer' file. (In cases where many sites discharge at once in the last statistical try, this may have to be adjusted manually.)

streamer2avs calls a routine which resets the coordinates of the cadmus cubic coordinates to those used by the AVS graphics system. It outputs a 'streamer-avs' file which must then be processed by "de2ucd streamer-avs". The latter performs the backward search as above, and restates the data in the ucd format, which enables AVS to pick it up rapidly.

The sample AVS network which is included, will then display the streamer in its cube of coordinates. (The outlines of the cube and needle are read in separately, and must be adjusted for the problem at hand). 


\section{$6 \quad$ Preparing graphics}

Copy the 'streamer' file from the current run (and make a permanent backup for later use.)

Examine the head end of the 'streamer' file. The Cadmus routines will not have listed the sites on the starting needle from which the first discharge links form their connection. These may be found by inspection, and added to the head of the list (with voltage 0.0000 and statistical trial number 0 , to fill the two right-hand columns.)

Examine the tail of the 'streamer' file. There will be one (or sometimes two)discharge sites in the plane adjacent to the cathode. These will show a voltage reading of $0.6^{* * *}$, considerably higher than the others because of the short remaining gap. One of these should be the last line in the file (either move it, or erase the lines which follow it), in order for nuldrread to trace the leader path back correctly.

Call "nuldrread". Call "wave", ".RUN xxx.pvw". PVWave will display a perspective white-on-black view of the tree. On Silicon Graphics machines, use snapshot and swapbw to obtain black-on-white. If Showcase is available, it is convenient for reshaping and printing.

Call "streamer2avs". Call "de2ucd streamer-avs" to form a similarnamed file with inp suffix. AVS can call the sample.net to display in its 3-D geometry viewer the cube and streamer, which will be color-banded to represent Monte-Carlo timing. Rotation and animated growth may be demonstrated. In the color-banded version, we do not display the heavy leader path for fixed printout. However, if one is doing an animated demonstration, it may be called from the 'ldr' file, above, as a separate display element in the last few frames.

\section{Routines for inclusion of diagonal links}

The cadmus routines in this 'diag/' subdirectory have been modified to include face-diagonal and body-diagonal links between sites on the cubic Cartesian lattice. This permits a wider choice of directional angle for each new link in the tree structure. Instead of the (somewhat rigid) restriction to 6 neighbor-directions, each growth tip now faces out to 12 "next-nearest" face-diagonal neighbor directions and 8 "next-next-nearest" body-diagonal neighbors.

Because these additional neighbors stand off at a greater separation from the existing tree (which is at zero potential), they will tend to have higher 
voltages (Laplacian- phi values) than the six "nearest" edge-diagonal neighbors. To weight their voltage values to an equivalent statistical level, we assume that the precise (3-D radial) Laplacian potential field in this "neighbor" or "active-growth" zone falls off inversely with the radius. The electric field ( which is the gradient of the potential and which instigates the discharge breakdown ) will then vary as the inverse square of the radius (or link-length). Thus, we down-weight the voltage of the face-diagonals by a factor of $1 / 2$, and the body-diagonals by $1 / 3$, before making the statistical comparison against weighted random numbers.

An overall result is to increase the a-priori probability of growth per statistical trial (Monte-Carlo time tick) by a ratio $6+(1 / 2 \mathrm{X} 12)+(1 / 3 \mathrm{X}$ $8)$ : 6 or roughly $2.444: 1$.

The augmented choice of angles at each step permits the fractal tree to grow with more freedom, even to the point of reversing direction.

The effect of selecting a higher exponent for the statistical weighting procedure, is to concentrate the growth likelihood from the tip towards the axial center of the solid angle visible to the tip, where the field strength is highest.

Ambiguity in the choice of path is resolved in favor of the shortest path. Thus, neighbor candidates alongside a "stem" or "trunk" of the fractal growth are not reached through a diagonal link, when a shorter diagonal or an edge connection is found for them.

Method: At each growth stage, the neighbors are chosen by combinations of dpcshifts on the existing tree. The path direction to each individual site is recorded by an integer on the corresponding site in the array 'intnbrs'. Integers 1-6 are used for the edge-neighbors; $7-18$ for the face-diagonal neighbors; and 19-26 for the body-diagonal neighbors. The order of choice eliminates ambiguities.

The array 'intnbrs' is passed to the statistical comparison routine, which now weights the Laplacian 'phi' values as described above, before making the statistical tests.

For each discharged link, the directional index integer is recorded in a separate column of the 'dgstreamer' file (note the change from 'streamer' label). The post-processing routine dgldrread can then read this file into the appropriate 'newtree' and 'ldr' files for graphical display as before.

\section{References}

[1] H. A. Fowler, J.E. Devaney, J.G. Hagedorn, and F.E. Sullivan, "Dielectric 
Breakdown in a Simplified Parallel Model", NISTIR No. 6174, June 1998.

[2] I. Beichl and F.E. Sullivan, "(Monte Carlo) Time after Time", Computational Science and Engineering 4, no. 3 (July-Sept. 1998), pp.91-94.

[3] D. Burns and R.B. Daoud, "Lam; an open cluster environment for mpi", in Supercomputing Symposium '94, June 1994, Toronto, Canada. Code available at http//www.osc.edu/lam.html.

[4] See http://www.mcs.anl.gov/Projects/mpi/mpich/index.html 


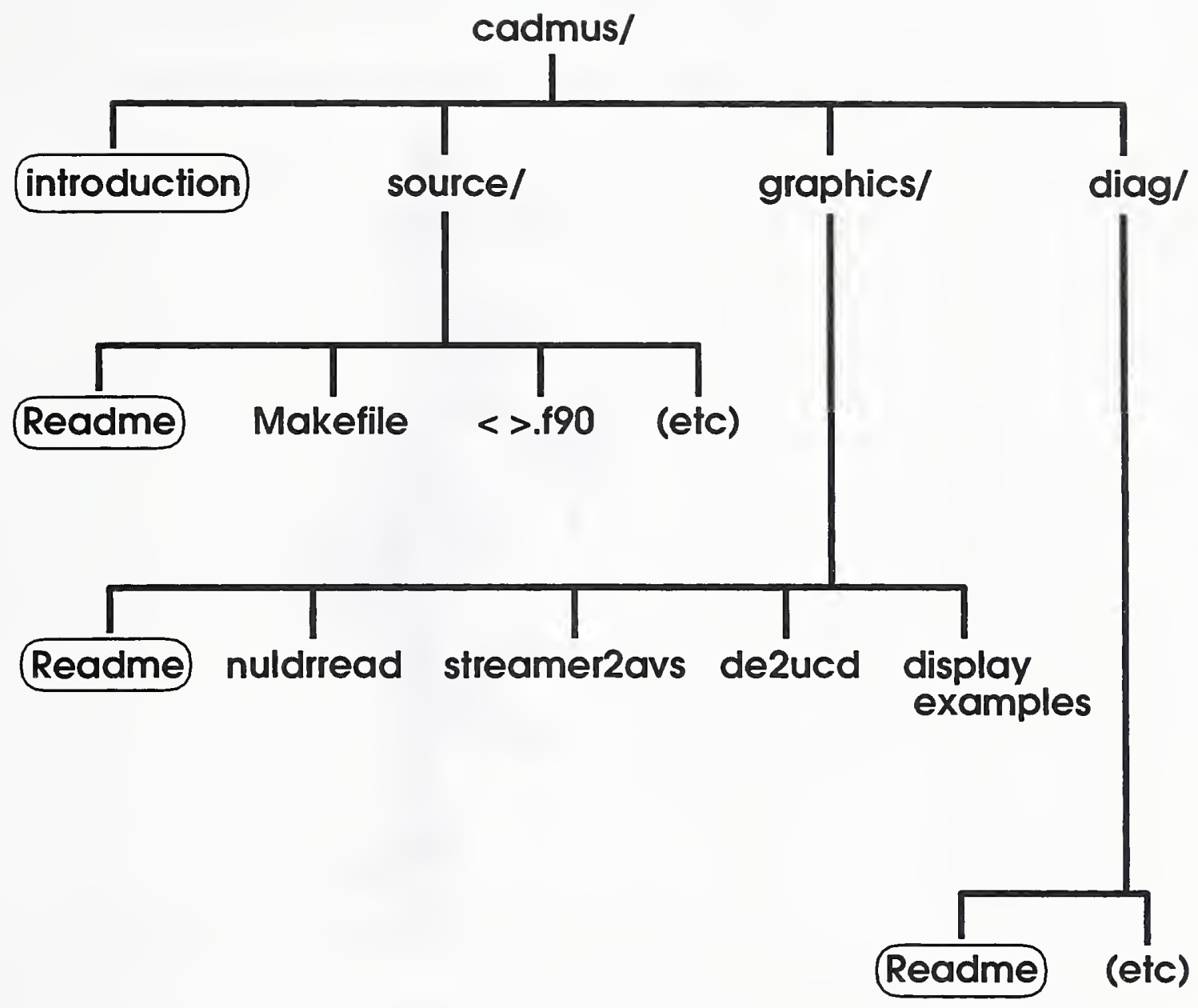

Figure 1: Cadmus Directories 


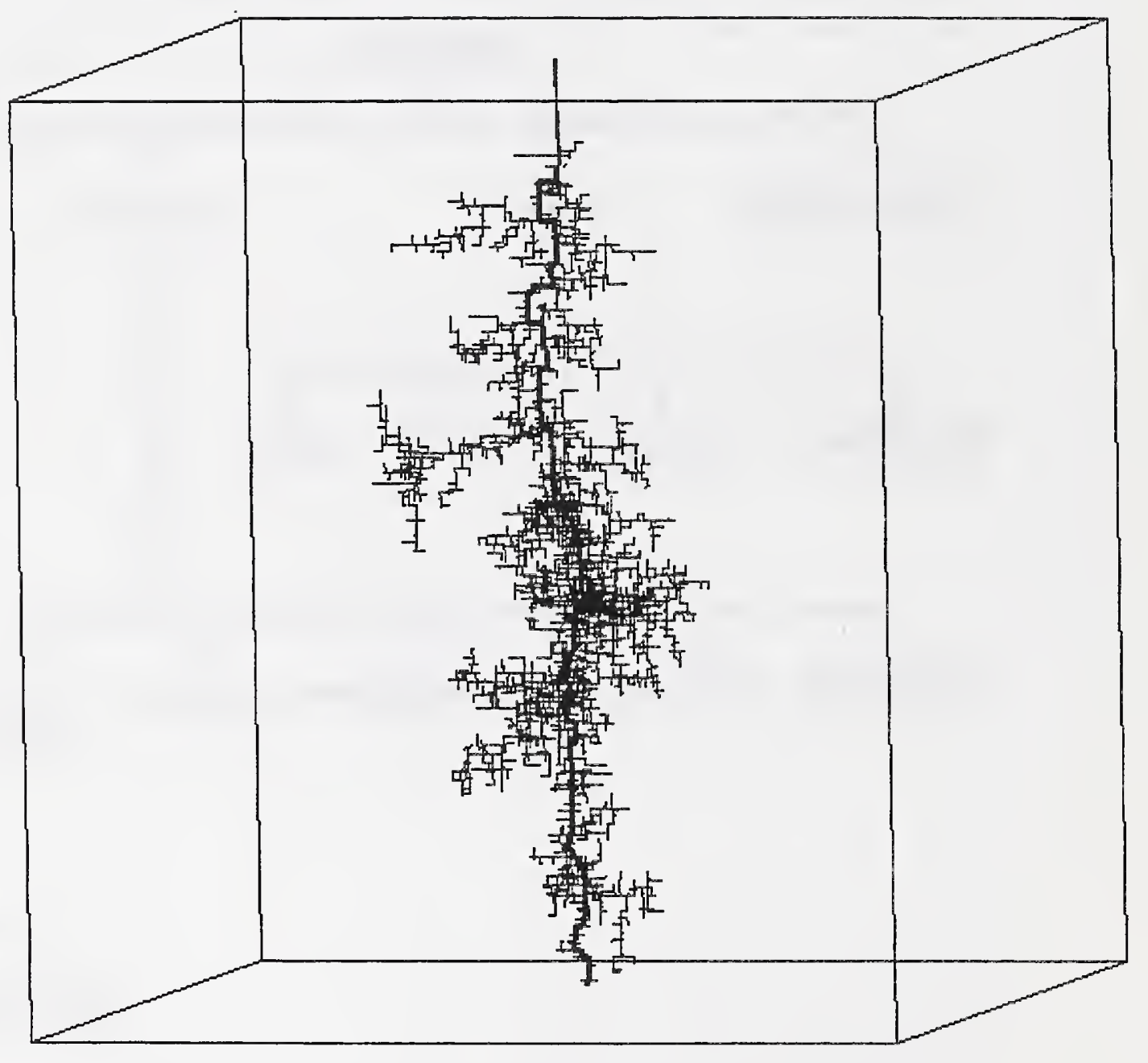

Figure 2: Sample of PVwave black-and-white graphics 

